\title{
Event-related potential abnormalities in schizophrenia: A failure to "gate in" salient information?
}

Colleen A. Brenner

Paul D. Kieffaber

William \& Mary

Brett A. Clementz

Brett A. Clementz

\section{Recommended Citation}

Brenner, C. A., Kieffaber, P. D., Clementz, B. A., Johannesen, J. K., Shekhar, A., O'Donnell, B. F., \& Hetrick, W. P. (2009). Event-related potential abnormalities in schizophrenia: a failure to "gate in" salient information?. Schizophrenia research, 113(2-3), 332-338.

This Article is brought to you for free and open access by the Arts and Sciences at W\&M ScholarWorks. It has been accepted for inclusion in Arts \& Sciences Articles by an authorized administrator of W\&M ScholarWorks. For more information, please contact scholarworks@wm.edu. 


\title{
Event-related potential abnormalities in schizophrenia: A failure to "gate in" salient information?
}

\author{
Colleen A. Brenner ${ }^{a, *}$, Paul D. Kieffaber ${ }^{b}$, Brett A. Clementz ${ }^{c}$, Jason K. Johannesen ${ }^{\text {d, }}$ \\ Anantha Shekhar ${ }^{\mathrm{e}, \mathrm{g}}$, Brian F. O'Donnell ${ }^{\mathrm{f,g}}$, William P. Hetrick ${ }^{\mathrm{f}, \mathrm{g}}$ \\ ${ }^{a}$ University of British Columbia, Department of Psychology, 2136 West Mall, Vancouver, B.C., Canada V6T IZ4 \\ ${ }^{b}$ College of William and Mary, Department of Psychology, 500 Landrum Drive, Williamsburg, VA 23185, USA \\ ' University of Georgia, Departments of Psychology and Neuroscience, Bioimaging Research Center, Psychology Building, Athens, GA 30602-3013, USA \\ d Yale University School of Medicine, Department of Psychiatry, VA Connecticut Health Care System, Psychology Service, 116-B, 950 Campbell Avenue, \\ West Haven, CT 06516, USA \\ e Indiana University School of Medicine, Department of Psychiatry, Institute of Psychiatric Research, 791 Union Drive, Indianapolis, IN 46202, USA \\ ${ }^{\mathrm{f}}$ Indiana University, Department of Psychological and Brain Sciences, 1101 East Tenth Street, Bloomington, Indiana 47405, USA \\ g Larue D. Carter Hospital, 2601 Cold Spring Road, Indianapolis, IN 46220, USA
}

\section{A R T I C L E I N F O}

\section{Article history:}

Received 7 April 2009

Received in revised form 10 June 2009

Accepted 12 June 2009

Available online 23 July 2009

\section{Keywords:}

Sensory gating

P50

Schizophrenia

ERP

Power

Salience

\begin{abstract}
A B S T R A C T
Sensory gating refers to the central nervous system's ability to filter sensory inputs, and can be measured by comparing the suppression of event-related brain potential (ERP) amplitudes in a paired auditory stimulus procedure. Poor gating scores in schizophrenia may be caused by abnormal responses to the first (S1), the second (S2) or both of the paired stimuli. However, since S1 and S2 responses may index separate psychological phenomenon, corresponding to the ability to "gate in" and "gate out" sensory stimuli respectively, the precise mechanism affected in schizophrenia remains unclear. To examine the extent to which saliency processing abnormalities may contribute to S1 response deficits, standard and rare (15\% probability) paired stimuli were presented to 21 participants with schizophrenia and 22 healthy controls. P50 and N100 ERP amplitude as well as low, beta and gamma frequency power were measured to examine the time course and relative contributions of oscillatory activity affecting auditory processing in schizophrenia. In this study, schizophrenia patients exhibited less evoked beta 1 power $(12-20 \mathrm{~Hz})$ in response to salient stimuli at S1, and lower $\mathrm{N} 100$ amplitude in response to all S1 stimuli. No group differences were found in the low, beta $2(20-30 \mathrm{~Hz})$, or gamma frequency ranges. These findings suggest aberrant sensory processing during stages of stimulus evaluation and saliency detection in schizophrenia.
\end{abstract}

(C) 2009 Elsevier B.V. All rights reserved.

\section{Introduction}

"Sensory gating" refers to the central nervous system's ability to filter sensory inputs. One method of assessing sensory

\footnotetext{
* Corresponding author. Tel.: +1 604822 4650; fax: +1 6048226923. E-mail addresses: cbrenner@psych.ubc.ca (C.A. Brenner), pdkieffaber@wm.edu (P.D. Kieffaber), clementz@uga.edu (B.A. Clementz), Jason.Johannesen@yale.edu (J.K. Johannesen), ashekhar@iupui.edu (A. Shekhar), bodonnel@indiana.edu (B.F. O'Donnell), whetrick@indiana.edu (W.P. Hetrick).
}

gating involves measuring mid-latency auditory evoked responses (MLAERs) to paired auditory stimuli. During this procedure, suppression of P50 amplitude to the second stimulus (S2) is ostensibly caused by recurrent inhibitory mechanisms activated by the first (S1) stimulus (Freedman et al., 1987). P50 magnitude of response is most often expressed as a ratio, with amplitude of the response to the second click divided by the amplitude of the response to the first click. Similarly, the N100 also exhibits a pattern of suppression to repeated stimuli. Schizophrenia subjects generally exhibit less sensory gating than healthy subjects for both of these MLAERs 
(Boutros et al., 2004; Clementz et al., 2003; Clementz, 1998; Clementz et al., 1997a; Patterson et al., 2008). Such findings are hypothesized to reflect neuronal gating deficits in schizophrenia that may cause sensory inundation and 'flooding' (Bunney et al., 1999); however much remains to be understood about the neuronal and cognitive mechanisms that influence sensory gating.

To this end, one important line of research aims to determine the factors that contribute to sensory gating scores among both healthy and pathological groups. One possibility is that gating is poor because S2 response amplitude is not suppressed compared to an otherwise normal S1 response. In this case, poor sensory gating indicates a failure of inhibitory neural mechanisms responsible for "gating out" redundant and irrelevant sensory stimuli (Clementz et al., 1997b; Freedman et al., 1987). A second pattern that leads to poor gating scores occurs when S1 response amplitude is abnormally small in the presence of an otherwise normal S2 response (Blumenfeld and Clementz, 2001; Clementz et al., 2003; Johannesen et al., 2005). One interpretation of this pattern is that poor gating is due to a failure of the nervous system to register and/or attend to the S1 stimulus. This conceptualization is consistent with the claim of Boutros and Belger (1999), that ERPs elicited in the paired stimuli procedure reflect the abilities of the nervous system to both (i) filter out extraneous information (as measured by ERP amplitude suppression at S2) and (ii) 'gate in' novel, or salient, information (as measured by ERP amplitude to S1). Interestingly, there is evidence for both "gating in" and "gating out" deficits in schizophrenia during the typical paired stimuli paradigm. However, whether MLAERs associated with "gating in" or "gating out" are differentially affected by manipulations of attention and expectancy (which are known to affect overall sensory gating scores) remains an important empirical question (Clementz et al., 2002; Cullum et al., 1993; Erwin et al., 1998; Guterman et al., 1992; Kogoj et al., 2005; Thoma et al., 2003; Woldorff et al., 1993).

Most sensory gating studies have relied on time domain ERP amplitude measurements. However, recent studies employing spectral frequency analyses have provided additional information about auditory sensory processing in schizophrenia. For instance, poor gating may, in part, reflect attenuated neural activation in response to S1 (i.e., "gating in" deficits) in the 1-20 Hz bandwidth (Blumenfeld and Clementz, 2001; Johannesen et al., 2005). Consistent with data demonstrating that beta activity reflects encoding, salience detection and consolidation of sensory information, Hong et al. (2008) found that beta activity in response to S1 predicted stronger gating and P50 suppression to S2 (Bibbig et al., 2001; Kisley and Cromwell, 2006; Hong et al., 2008). Neural activity in the gamma frequency range, previously associated with immediate sensory registration, contributes to the morphology of the P50 ERP component (Kopell et al., 1999; Clementz and Blumenfeld, 2001), but the findings regarding the contribution of gamma activity to auditory sensory gating deficits in schizophrenia are unclear. Some studies have failed to find differences in the gamma band response between healthy and schizophrenia participants (Clementz and Blumenfeld, 2001; Clementz et al., 1997a), whereas others have reported decreased gamma and low frequency responses to S1 in schizophrenia (Johannesen et al., 2005) as well as a selective reduction in gamma band suppression in association with higher ratings of subjective perceptual disturbance (Johannesen et al., 2008). Therefore, it is important to further evaluate the frequency composition of EEG activity elicited by manipulations of the paired stimuli paradigm. Given the emerging data on the functional significance of oscillatory activity within specific frequency ranges (Deiber et al., 2007; Kisley and Cromwell, 2006; Klimesch, 1999; Krishnan et al., 2005; Palva and Palva, 2007; Pantev et al., 1991; Pesonen et al., 2007; Schack et al., 2005; Tallon-Baudry, 2003; Trautner et al., 2006; Whittington et al., 1997), it is likely that assessing activity in these bands will provide information about the underlying neurophysiological and cognitive deficits in those with schizophrenia.

Therefore, the primary aims of the present study were to characterize the frequency composition of the MLAER electrophysiological response and explicate the nature of the differences between schizophrenia and healthy subjects with respect to the frequency composition of responses at S1 and S2 in the paired stimuli paradigm. First, we examined the effects of stimulus salience on MLAER responses, and predicted that (i) salient stimuli (infrequent paired clicks of a deviant pitch) would augment evoked response amplitudes across all participants, but (ii) schizophrenia subjects would have less augmentation than controls to rare stimuli. Second, we sought to determine whether differences in schizophrenia are better explained by difficulty "gating in" salient sensory information at S1 or to difficulty "gating out" redundant sensory information at S2, and predicted that (iii) schizophrenia subjects would have "gating in" deficits as indicated by reduced S1 amplitudes to both frequent and rare stimuli. Third, we examined which frequency band best accounted for S1 and gating deficits in schizophrenia, and predicted, based on previously reported associations between beta frequency activity and salient stimulus processing, that (iv) low beta $(12-20 \mathrm{~Hz})$ frequency information would best account for stimulus-driven salience effects. These finding provide a more comprehensive characterization of the neurophysiological and cognitive processes that best account for poor auditory gating in schizophrenia.

\section{Materials and methods}

\subsection{Participants}

The participants were 21 individuals ( 3 females, mean age $=36, \mathrm{SD}=13$ ) meeting criteria for schizophrenia $(N=17)$ or schizoaffective disorder $(N=4)$ based on the Structured Clinical Interview for DSM-IV (1994) and 22 healthy persons ( 6 females, mean age $=38, S D=11$ ) free from any Axis I or Axis II disorders (ratings done by authors $C$. A.B. and A.S.). None of the participants met criteria for alcohol dependence within the past 2 years or substance abuse within 6 months prior to testing. Exclusion criteria included head injury, learning disability, hearing impairment, verbal IQ less than 75, and alcohol or illicit substance use within $24 \mathrm{~h}$ prior to testing. The number of individuals who smoked cigarettes within $24 \mathrm{~h}$ of testing did not differ between groups, and participants were not permitted to smoke during the 40 min preceding testing, thus minimizing possible acute 
effects of nicotine on ERP amplitudes (Adler et al., 1993). Findings reported below were unchanged when smoking status was used as a statistical covariate.

All but one patient with schizophrenia was taking antipsychotic medication (2 typical, 18 atypical), thus group size was too small for statistical comparison between medication subgroups. There were no significant correlations between any electrophysiological variable and chlorpromazine equivalent dosage.

\subsection{Stimuli and procedures}

One-hundred and thirty paired stimuli were presented binaurally while participants were seated in a sound attenuated room. Stimuli were two different paired auditory sounds: (1) standard click pairs ( $85 \%$ probability; $N=110$ trials, 3 ms duration, peak intensity $81 \mathrm{~dB}$ SPL as measured by a Simpson Model 886 sound level meter, fast mode, "A" weighting, $\mathrm{SOA}=500 \mathrm{~ms}$ ) and (2) rare $1000 \mathrm{~Hz}$ paired tone pips ( $15 \%$ probability, $3 \mathrm{~ms}$ duration, rise/fall of $.75 \mathrm{~ms}$, peak intensity $81 \mathrm{~dB}$ SPL, SOA $=500 \mathrm{~ms}$ ). Inter-trial interval varied randomly between 7 and $11 \mathrm{~s}$ (mean $=9 \mathrm{~s}$ ). Stimuli were presented against a $58 \mathrm{~dB}$ SPL white-noise background. To keep all participants alert, subjects were asked to respond by button press to the rare paired tone pips.

\subsection{EEG recordings and data processing}

Electroencephalographic activity was collected from 29 recording sites $(1000 \mathrm{~Hz}$ A/D rate; $0.10 \mathrm{~Hz}$ high pass, $200 \mathrm{~Hz}$ low pass; gain $=10 \mathrm{~K}$; nose reference; impedances $\leq 10 \mathrm{k} \Omega$ ) using sintered $\mathrm{Ag}-\mathrm{AgCl}$ electrodes.

\subsubsection{ERP peak measurement}

P50 responses at $\mathrm{Cz}$ were segmented ( -50 to $400 \mathrm{~ms}$ ), baseline corrected, ocular artifacts were corrected (Gratton et al., 1983), and remaining trials with values exceeding $\pm 100 \mu \mathrm{V}$ were excluded from analyses. A 1$50 \mathrm{~Hz}$ ( $24 \mathrm{~dB} /$ octave) bandpass filter was applied to averaged data and peak amplitude was defined as the difference in $\mu \mathrm{V}$ between the most positive point 40 to $80 \mathrm{~ms}$ after stimulus onset and the point of maximum deflection on the preceding negative trough (Fig. 1).
Responses to the second click were measured within $\pm 15 \mathrm{~ms}$ of the response to the first click. The amplitude of the N100 response was defined as the most negative point 80 to $150 \mathrm{~ms}$ after stimulus onset (referenced to baseline).

\subsubsection{Spectral frequency power}

Frequency bands of interest were identified as low (1$12 \mathrm{~Hz})$, beta $1(12-20 \mathrm{~Hz})$, beta $2(20-30 \mathrm{~Hz})$ and gamma (30-50 Hz). We did not further subdivide the low frequency band because the ISI of $500 \mathrm{~ms}$ does not allow for reliable measurement of low frequency activity. Raw EEG recordings were segmented into $700 \mathrm{~ms}$ epochs ( $250 \mathrm{~ms}$ baseline), and subjected to the same ocular correction and artifact rejection criteria as the peak amplitude analyses. The time-varying spectral power, $W_{x}=|\omega(t, f) * s(t)|^{2}$ was computed for each frequency $(f)$ by convolving the average ERP signal, $s(t)$, with a complex-valued Morlet's wavelet, $\omega(t ; f)$,

$\omega(t, f)=A \cdot e^{2 i \pi f t} \cdot e^{-t^{2} / 2 \sigma_{t}^{2}}$

where $\sigma_{\mathrm{f}}=1 /\left(2 \pi \sigma_{\mathrm{t}}\right)$, and $A=\sigma_{t}^{-1} \cdot(2 / \pi)^{1 / 2}$ is a Gabor normalization factor (Tallon-Baudry and Bertrand, 1999). In the context of Eq. (1), A yields the instantaneous amplitude (Hermann et al., 2005, p. 240). The family of wavelets used were characterized by the constant ratio $f / \sigma_{\mathrm{f}}=7$. Signal power was computed over the range of frequencies from 1 to $70 \mathrm{~Hz}$ in $1 \mathrm{~Hz}$ steps.

To identify the latency of the peak power within each frequency band, the raw EEG was segmented from -100 to $500 \mathrm{~ms}$ and averaged across S1, S2, standard and rare trials for each frequency band separately. The 'frequency extraction' procedure in Brain Vision Analyzer software (Brain Products, Munich, Germany) converted the data into power within a specified frequency range at each time point. We then measured the latency at which the power peaked for each subject, and used that average ( \pm 1.5 standard deviations) to define the region that we analyzed our wavelet-based timefrequency data. This approach allowed us to define the window used in our time-frequency analysis based on the points in which activity within that frequency band was largest. For low frequency data the analysis window spanned from 118-310 ms; for beta 1 activity it was 20-167 ms; for
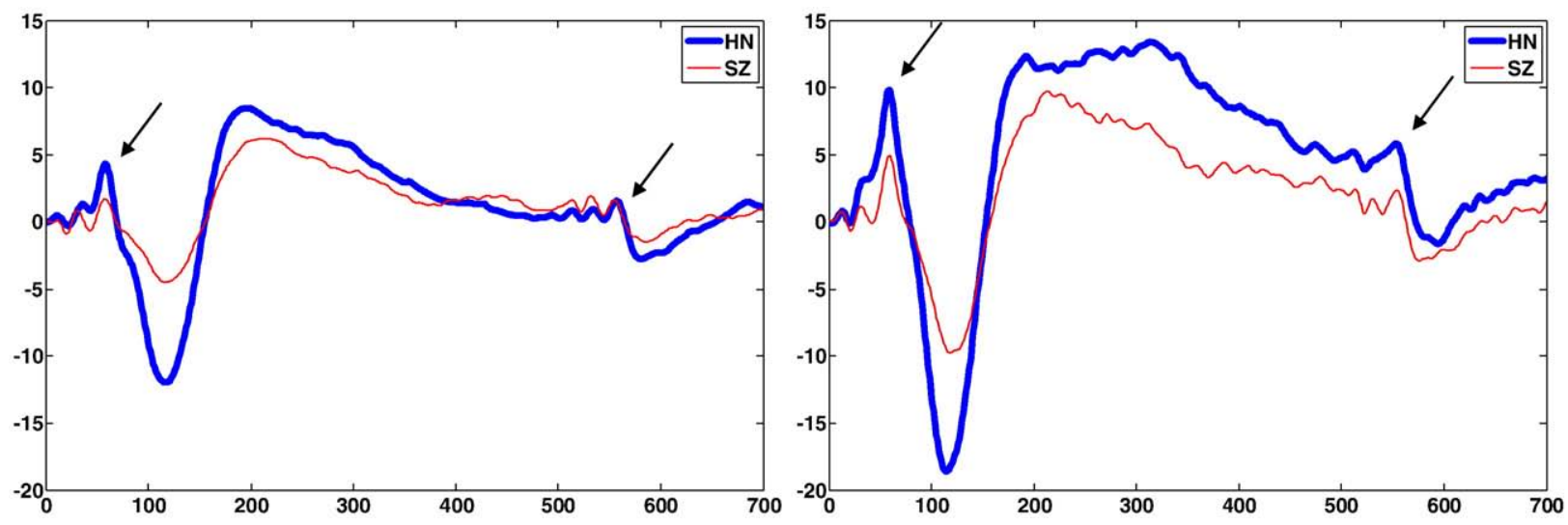

Fig. 1. ERP waveforms (1-50 Hz filter) for each diagnostic group. The left panel shows the waveforms in response to 'standard' stimuli and the right panel shows the waveforms in response to 'rare' stimuli. 
beta 2 activity it was 23-157 ms; and for gamma it was 32$74 \mathrm{~ms}$.

\subsection{Statistical analyses}

A square root transformation was applied to the P50 peak amplitude data to better approximate a normal distribution. Statistical outliers were determined as participants with values at least three times the interquartile range of the corresponding participant group (Brenner et al., 2004), and were eliminated from further analyses. There were no more than six outliers in a given analysis (three $\mathrm{Sz}$ and three controls). Difference scores were computed across click stimuli (S1-S2), rather than the S2/ S1 ratio, because it is a more psychometrically reliable index of P50 evoked response attenuation (Smith et al., 1994). Within and between-group contrasts were conducted using Repeated measures ANOVA, with Diagnosis (SZ, HC) as the betweengroup factor and Stimulus (S1, S2) and Salience (rare, standard) as within-group factors.

\section{Results}

\subsection{Behavioral responses}

Button-press responses to the presentation of the rare stimuli did not differ between groups (control $=98.6 \%$ correct; $\mathrm{SD}=.03$; schizophrenia $=95.7 \%$ correct; $\mathrm{SD}=.06)$.

\subsection{P50 ERPS}

Repeated Measures ANOVA yielded: a main effect of Salience, $F(1,37)=32.8, p=.001, \eta_{\mathrm{p}}^{2}=.47$, indicating larger responses to rare compared to standard paired stimuli; a main effect of Stimulus, $F(1,37)=71.9, p=.001, \eta_{\mathrm{p}}^{2}=.66$, indicating larger responses to S1 than to S2; and a Salience $\times$ Stimulus interaction, $F(1,37)=11.2, p=.002, \eta_{\mathrm{p}}^{2}=.23$, reflecting greater S1 amplitude to rare compared to standard stimuli for both groups (Table 1). For the S1-S2 amplitude difference score, there was a main effect of Salience, $F(1,39)=12.2$, $p=.001, \eta_{\mathrm{p}}^{2}=.24$, indicating a larger difference between $\mathrm{S} 1$ and $\mathrm{S} 2$ in response to rare compared to standard paired stimuli for both groups ${ }^{1}$. Repeated measures ANOVAs were repeated using only those 'standard' trials that immediately preceded a rare trial, thereby ensuring the same number of trials contributed to the average waveform for both trial types. These analyses resulted in a main effect of Stimulus, $F$ $(1,36)=26.1, p=.001, \eta_{\mathrm{p}}^{2}=.42$, and a Salience $\times$ Stimulus interaction, $F(1,36)=15.8, p=.001, \eta_{\mathrm{p}}^{2}=.31$, when using S1 and S2 amplitudes. Results using the S1-S2 difference score did not change when this procedure was applied. There were no group differences in P50 gating.

\subsection{N100 ERPS}

Repeated Measures ANOVA revealed: a main effect of Salience, $F(1,40)=118.7, p=.001, \eta_{\mathrm{p}}^{2}=.75$, indicating larger responses to rare compared to standard paired stimuli; a main

\footnotetext{
1 Analysis of the P50 responses and difference score in response to frequent stimuli using different filter parameters $(10-50 \mathrm{~Hz})$ also did not yield significant group differences.
}

Table 1

Untransformed ERP amplitudes (in $\mathrm{uV}$ ) and power values (in $\mathrm{uV}^{2}$ ).

\begin{tabular}{|c|c|c|c|c|}
\hline & S1 M (SD) & S2 M (SD) & S1-S2 M (SD) & $\mathrm{S} 2 / \mathrm{S} 1$ (SD) \\
\hline \multicolumn{5}{|c|}{ P50 ERP } \\
\hline \multicolumn{5}{|c|}{ Frequent } \\
\hline $\mathrm{HN}$ & $4.62(2.58)$ & $1.55(.89)$ & $2.97(2.47)$ & $.94(2.45)$ \\
\hline SZ & $4.28(2.46)$ & $2.35(2.11)$ & $1.94(3.14)$ & $.73(.71)$ \\
\hline \multicolumn{5}{|c|}{ Rare } \\
\hline HN & $7.97(4.23)$ & $2.86(2.46)$ & $5.34(4.63)$ & $.39(.36)$ \\
\hline SZ & $8.01(4.72)$ & $2.92(2.36)$ & $5.09(4.58)$ & $.50(.45)$ \\
\hline \multicolumn{5}{|c|}{ N100 ERP } \\
\hline \multicolumn{5}{|c|}{ Frequent } \\
\hline $\mathrm{HN}$ & $-14.51(6.76)$ & $-3.25(3.19)$ & $-11.25(5.40)$ & $.200(.16)$ \\
\hline SZ & $-8.93(3.91)$ & $-2.77(1.90)$ & $-6.16(3.78)$ & $.337(.30)$ \\
\hline \multicolumn{5}{|c|}{ Rare } \\
\hline HN & $-24.54(10.66)$ & $-5.99(3.81)$ & $-18.550(9.57)$ & $.215(.13)$ \\
\hline SZ & $-16.14(8.40)$ & $-5.11(3.96)$ & $-11.028(8.33)$ & $.281(.34)$ \\
\hline \multicolumn{5}{|c|}{ Low power (1-12 Hz) } \\
\hline \multicolumn{5}{|c|}{ Frequent } \\
\hline HN & $4.78(5.07)$ & $1.56(2.82)$ & $3.22(6.11)$ & \\
\hline SZ & $3.69(3.66)$ & $1.98(2.82)$ & $1.71(5.28)$ & \\
\hline \multicolumn{5}{|c|}{ Rare } \\
\hline HN & $5.98(5.39)$ & $.73(1.12)$ & $5.26(5.68)$ & \\
\hline SZ & $4.28(1.90)$ & $1.37(1.83)$ & $2.91(2.70)$ & \\
\hline \multicolumn{5}{|c|}{ Beta 1 power $(12-20 \mathrm{~Hz})$} \\
\hline \multicolumn{5}{|c|}{ Frequent } \\
\hline HN & $2.94(4.78)$ & $1.92(3.63)$ & $1.01(5.85)$ & \\
\hline SZ & $3.13(3.36)$ & $1.55(3.74)$ & $1.58(5.32)$ & \\
\hline \multicolumn{5}{|c|}{ Rare } \\
\hline HN & $9.31(7.76)$ & $1.74(2.76)$ & $7.57(8.60)$ & \\
\hline SZ & $4.03(4.54)$ & $1.44(1.79)$ & $2.59(4.49)$ & \\
\hline \multicolumn{5}{|c|}{ Beta 2 power $(20-30 \mathrm{~Hz})$} \\
\hline \multicolumn{5}{|c|}{ Frequent } \\
\hline $\mathrm{HN}$ & $2.34(4.43)$ & $1.90(3.61)$ & $.439(4.11)$ & \\
\hline SZ & $2.65(3.37)$ & $2.02(4.28)$ & $.628(5.22)$ & \\
\hline \multicolumn{5}{|c|}{ Rare } \\
\hline HN & $3.47(4.05)$ & $2.67(2.76)$ & $.800(3.81)$ & \\
\hline SZ & $3.79(4.52)$ & $1.32(2.00)$ & $2.47(3.91)$ & \\
\hline \multicolumn{5}{|c|}{ Gamma Power $(30-50 \mathrm{~Hz})$} \\
\hline \multicolumn{5}{|c|}{ Frequent } \\
\hline $\mathrm{HN}$ & $5.52(7.79)$ & $2.64(6.21)$ & $2.88(7.57)$ & \\
\hline SZ & $2.98(5.78)$ & $1.14(2.18)$ & $1.84(5.67)$ & \\
\hline \multicolumn{5}{|c|}{ Rare } \\
\hline HN & $7.38(10.76)$ & $2.12(3.68)$ & $5.26(8.91)$ & \\
\hline SZ & $6.28(12.83)$ & $2.30(4.22)$ & $3.98(9.22)$ & \\
\hline
\end{tabular}

effect of Stimulus, $F(1,40)=137.3, p=.001, \eta_{\mathrm{p}}^{2}=.77$, indicating larger responses to S1 than S2; and a Stimulus $\times$ Diagnosis interaction, $F(1,40)=9.9, p=.003, \eta_{\mathrm{p}}^{2}=.20$, reflecting greater S1 amplitude by the healthy control group. Finally, there was a Salience $\times$ Stimulus interaction, $F(1,40)=40.7, p=.001$, $\eta_{\mathrm{p}}^{2}=.51$, indicating greater $\mathrm{S} 1$ amplitude to rare compared to standard stimuli for both groups. For the S1-S2 difference scores, there was a main effect of Salience, $F(1,40)=40.7$, $p=.001, \eta_{\mathrm{p}}^{2}=.51$, indicating a larger difference between $\mathrm{S} 1$ and S2 in response to rare compared to standard paired stimuli for both groups.

\subsection{Time frequency analyses}

Repeated Measures ANOVA compared groups on power values for each frequency band separately (see Table 1). 
Within the low frequency $(1-12 \mathrm{~Hz})$ range, there was a main effect of Stimulus, $F(1,36)=41.94, p=.001, \eta_{p}^{2}=.54$, that indicated greater power in response to S1 compared to S2 (Fig. 2). There were no significant findings using the difference scores.

Within the beta 1 frequency range $(12-20 \mathrm{~Hz})$, analyses revealed a main effect of Salience, $F(1,36)=13.31, p=.001$, $\eta_{\mathrm{p}}^{2}=.27$, indicating greater beta power in response to rare stimuli, and a main effect of Stimulus, $F(1,36)=21.44$, $p=.001, \eta_{\mathrm{p}}^{2}=.37$, that indicated greater power in response to $\mathrm{S} 1$ compared to $\mathrm{S} 2$. A Salience $\times$ Diagnosis interaction, $F$ $(1,36)=5.92, p=.020, \eta_{\mathrm{p}}^{2}=.14$, indicated less beta 1 power in schizophrenia than in controls in response to rare stimuli. A Salience $\times$ Stimulus interaction, $F(1,36)=9.48, p=.004$, $\eta_{\mathrm{p}}^{2}=.21$, indicated greater beta 1 power in response to rare S1 stimuli. Finally, there was a Salience $\times$ Stimulus $\times$ Diagnosis interaction, $F(1,36)=11.41, p=.002, \eta_{\mathrm{p}}^{2}=.24$. To better understand this interaction, separate analyses were performed in response to standard and rare stimuli. For standard stimuli there was a main effect of Stimulus, $F(1,36)=$ 4.93, $p=.033, \eta_{\mathrm{p}}^{2}=.12$, that indicated greater power in response to $\mathrm{S} 1$. For rare stimuli there was a main effect of Stimulus, $F(1,36)=21.36, p=.001, \eta_{\mathrm{p}}^{2}=.37$, and a significant Stimulus $\times$ Diagnosis interaction, $F(1,36)=5.48$, $p=.025, \eta_{\mathrm{p}}^{2}=.13$, that indicated lower beta 1 power in response to $\mathrm{S} 1$ to rare stimuli by the schizophrenia group. For the S1-S2 difference scores, there was a main effect of Salience, $F(1,40)=10.89, p=.002, \eta_{\mathrm{p}}^{2}=.21$, and a Salience $\times$ Diagnosis interaction, $F(1,40)=5.96, p=.019, \eta_{p}^{2}=.13$. These findings indicated less change in beta 1 power from S1 to S2 in response to rare stimuli pairs in schizophrenia (Fig. 2).

Within the beta 2 frequency range (20-30), analyses revealed a main effect of Salience, $F(1,34)=8.80, p=.005$, $\eta_{\mathrm{p}}^{2}=.21$, that indicated greater beta 2 power in response to rare stimuli, and a main effect of Stimulus, $F(1,34)=6.36$, $p=.017, \eta_{\mathrm{p}}^{2}=.16$, indicating greater power in response to S1 compared to S2 (Fig. 2). There were no significant findings using the difference scores.

Gamma frequency power analyses revealed a marginally significant main effect of Salience, $F(1,38)=4.06, p=.051$, $\eta_{\mathrm{p}}^{2}=.10$, indicating greater gamma power in response to rare compared to standard stimuli, and a main effect of Stimulus, $F$ $(1,38)=12.79, p=.001, \eta_{\mathrm{p}}^{2}=.25$, indicating greater gamma power in response to S1 compared to S2 (Fig. 2). There were no significant findings using the difference scores.

\subsection{Time frequency and N100 amplitude correlations}

Bivariate correlations were performed between beta 1 power and N100 amplitude for each group separately. Within the control group N100 amplitude at S1 in response to rare stimuli was significantly correlated with beta 1 power at S1 in response to rare stimuli $(r=.467, p=.044)$. Within the schizophrenia group N100 amplitude at S1 in response to frequent stimuli was significantly correlated with both beta power at $\mathrm{S} 1$ in response to frequent $(r=.536, p=.018)$ and rare stimuli $(r=.567, p=.011)$. In addition, within the schizophrenia group N100 amplitude difference score to frequent stimuli was negatively associated with beta 1 power at $\mathrm{S} 1$ in response to rare stimuli $(r=-.600, p=.008)$.

\section{Discussion}

Individuals with schizophrenia exhibited less neural activity in the beta 1 frequency range (12-20 Hz) in response to rare, more salient stimuli, an effect that was driven by the low magnitude of their response to the initial stimulus in a dual-click procedure. The N100 analyses revealed a similar pattern of smaller S1 amplitudes in the schizophrenia group resulting in schizophrenia patients having less N100 "gating"

\section{$\mathrm{HC}$}

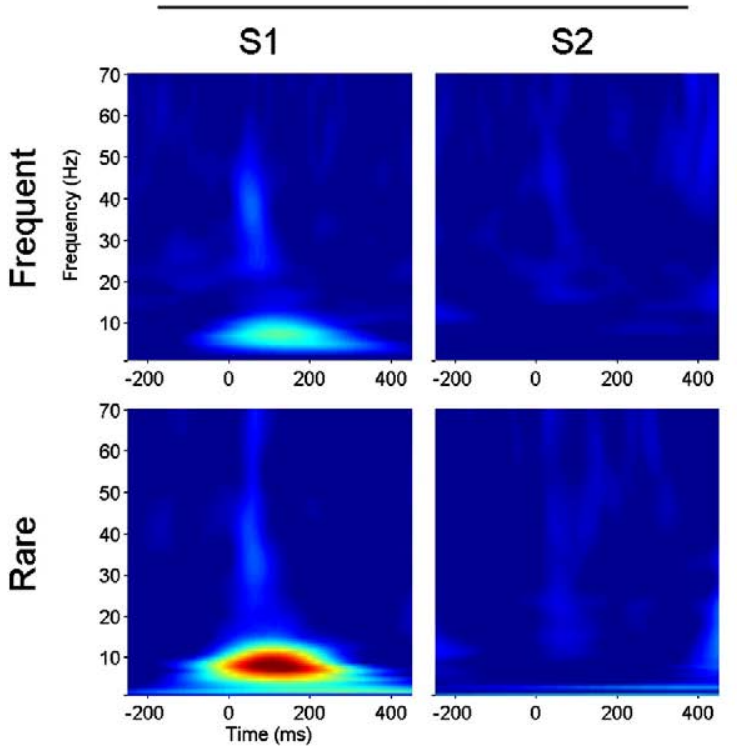

$S Z$
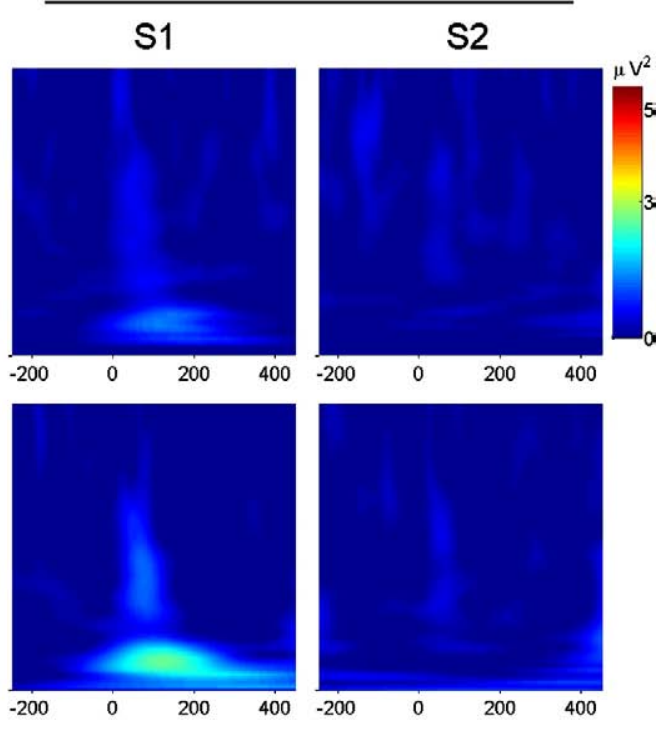

Fig. 2. Wavelet-based time-frequency transforms of the averaged ERP waveform. The upper panels show power over time in response to standard stimuli, and the lower panels show power over time in response to rare stimuli. Data from the healthy control group is shown on the left (S1 and S2 in columns) and the data from the schizophrenia group is shown on the right (S1 and S2 in columns). Patients demonstrated reduced beta 1 power (12-20 Hz) in response to rare S1 stimuli. 
than the healthy controls, although this finding was not specific to rare stimuli. Correlations between N100 amplitude and beta 1 activity reflect a relationship between these two measures; with N100 amplitudes to S1 (both frequent and rare stimuli) positively correlated with beta 1 power to S1 (both frequent and rare stimuli). Interestingly, the saliencedriven deficits in schizophrenia were not seen in the low (1$12 \mathrm{~Hz}$ ), beta $2(20-30 \mathrm{~Hz})$ or gamma frequency $(30-50 \mathrm{~Hz})$ range, and P50 amplitude gating did not differ between groups. Taken together, the present findings suggest a possible functional differentiation between sensory registration and mechanisms associated with sensory processing during stages of stimulus evaluation and saliency detection that may be dysfunctional in schizophrenia (Clementz et al. 2008).

In particular, our finding that schizophrenia patients exhibited less S1 beta 1 power to rare stimuli may indicate deficits in the stimulus evaluation stage of sensory encoding. This deficit, along with sensory gating deficits in the later stages of information processing (N100 and P200), is consistent with a problem in the nervous system's ability to detect and respond to salient or important information ("gating in") in schizophrenia (Boutros et al. 2004). In addition, increased stimulus saliency as manipulated by ISI (Kisley and Cromwell, 2006) and/or expectancy (Clementz et al., 2002) has been associated with increased beta activity in healthy controls, while less beta activity in response to S1 has been associated with aberrant sensory gating scores in healthy controls and patients with schizophrenia (Hong et al., 2004; Hong et al., 2008). Finally, in-vitro hippocampal stimulation studies suggest that beta oscillations may reflect encoding and evaluation of a percept (Haenschel et al., 2000; Hong et al., 2004; Traub et al., 1999). Taken together, these findings suggest that oscillatory activity in the beta range represents a measure of stimulus-driven salience detection that is altered in schizophrenia. While it is impossible to disentangle the effect of salience alone from the different physical characteristics of the rare stimuli that make it salient, this issue does not nullify the group differences that are the main finding of this report.

P50 ERP amplitude was larger to rare compared to standard paired stimuli, indicating sensitivity to salience. However, the P50 gating score did not significantly differ between groups. This finding, along with that of the gamma band results, may indicate intact sensory registration in patients with schizophrenia. There are, however, several alternative interpretations of this result including: (1) the range of P50 gating scores across studies is large and may be related to a number of methodological factors (Blumenfeld and Clementz, 2001; Bunney et al., 1999); (2) the effect size of our P50 peak-picked results is small compared to those of our N100 and frequency band results, suggesting lower discriminative power in P50; (3) reductions in P50 and evoked gamma responses may reflect pathophysiology that is present in only select subgroups of patients with schizophrenia (Johannesen et al., 2008); (4) the atypical medications taken by the majority of the patients in this study may further reduce the schizophrenia-normal effect size differences (Adler et al., 2004; Light et al., 2000); and (5) the use of rare tone pairs in a button-press target detection procedure, interspersed within the standard click pairs, may have altered the fundamental nature of the dual-click procedure. Future studies that clarify these issues, using larger sample sizes, are warranted.

In summary, previous investigations have identified at least two stages of auditory processing: stimulus registration (i.e., encoding that a stimulus has been presented) and evaluation (i.e., classifying the nature of the stimulus) (Boutros and Belger, 1999). There is evidence to suggest that neural oscillatory activity within the gamma and beta frequency ranges, respectively, may support these functions. The current study evaluated these functions in patients with schizophrenia using a modified dual-click procedure. We found that individuals with schizophrenia exhibited reduced N100 amplitude in response to the initial click stimulus and reduced beta 1 activity $(12-20 \mathrm{~Hz}$ ) in response to the initial salient stimulus. Patients did not significantly differ from healthy comparison subjects in P50 amplitude, low, beta 2 or gamma activity. These findings extend previous research by investigating the difference in neural response to both standard and salient, or rare, paired stimuli within a more detailed frequency band analysis than has previously been reported in the literature (Blumenfeld and Clementz, 2001; Johannesen et al., 2005). While P50 ERP amplitudes were increased to rare stimuli in both groups, beta 1 activity (12$20 \mathrm{~Hz}$ ), which is associated with stimulus saliency and evaluation, exhibited sensitivity to diagnosis. These findings suggest that aberrant stimulus evaluation, especially as it relates to stimulus salience detection, may be considered a primary mechanism underlying sensory processing-related EEG abnormalities in schizophrenia.

\section{Role of funding source}

Funding for this study was provided by NIMH grant MH074983-0 WPH. The NIMH had no further role in study design; in the collection, analysis and interpretation of data; in the writing of the report; and in the decision to submit the paper for publication.

\section{Contributors}

Dr. Brenner supervised data collection, processed and analyzed the ERP data and wrote the first draft of the manuscript. Dr. Kieffaber performed the time-frequency analysis of the data, created figures and edited the manuscript. Dr. Clementz contributed to the initial study design and edited the manuscript. Dr. Johannesen supervised data collection and edited the manuscript. Dr. Shekhar provided diagnostic and clinical information for the patients that participated in this study. Dr. O'Donnell was involved with study design, supervision of data collection and edited the manuscript. Dr. Hetrick was primarily responsible for study design and implementation, with supervision of data processing and analysis and edited the manuscript. All authors contributed to and have approved the final manuscript.

\section{Conflict of interest}

The authors have no conflicts of interest to report.

\section{Acknowledgements}

We would like to thank the participants for their contributions to this project and the research assistants that helped in the collection of this data.

\section{References}

Adler, L., Hoffer, L., Wiser, A., Freedman, R., 1993. Normalization of auditory physiology by cigarette smoking in schizophrenic patients. Am. J. Psychiatry 150 (12), 1856-1861.

Adler, L.E., Olincy, A., Cawthra, E., McRae, K., Harris, J., Nagamoto, H., et al. 2004. Varied effects of atypical neuroleptics on P50 auditory gating in schizophrenia patients. Am. J. Psychiatry 161 (10), 1822-1828. 
Bibbig, A., Faulkner, H.J., Whittington, M.A., Traub, R.D., 2001. Self-organized synaptic plasticity contributes to the shaping of gamma and beta oscillations in vitro. J. Neurosci. 21 (22), 9053-9067.

Blumenfeld, L., Clementz, B., 2001. Response to the first stimulus determines reduced auditory evoked response suppression in schizophrenia: single trial analysis using MEG. Clin. Neurophysiol. 112 (9), 1650-1659.

Boutros, N., Belger, A., 1999. Midlatency evoked potentials attenuation and augmentation reflect different aspects of sensory gating. Biol. Psychiatry 45, 917-922.

Boutros, N.N., Korzyukov, O., Jansen, B., Feingold, A., Bell, M., 2004. Sensory gating deficits during the mid-latency phase of information processing in medicated schizophrenia patients. Psychiatry Res. 126, 203-215.

Brenner, C., Edwards, C., Carroll, C., Kieffaber, P., Hetrick, W., 2004. P50 and acoustic startle gating are not related in healthy participants. Psychophysiology 41 (5), 702-708.

Bunney, W.E., Hetrick, W.P., Bunney, B.G., Patterson, J.V., Jin, Y., Potkin, S.G., et al., 1999. Structured interview for assessing perceptual anomalies (SIAPA). Schizophr. Bull. 25 (3), 577-592.

Clementz, B.A., 1998. Psychophysiological measures of (dis)inhibition as liability indicators for schizophrenia. Psychophysiology 35, 648-668.

Clementz, B.A., Blumenfeld, L.D., Cobb, S., 1997a. The gamma band response may account for poor P50 suppression in schizophrenia. NeuroReport 8 , 3889-3893.

Clementz, B.A., Geyer, M.A., Braff, D.L., 1997b. P50 suppression among schizophrenia and normal comparison subjects: a methodological analysis. Biol. Psychiatry 41, 1035-1044.

Clementz, B.A., Blumenfeld, L.D., 2001. Multichannel electroencephalographic assessment of auditory evoked response suppression in schizophrenia. Exp. Brain Res. 139, 377-390.

Clementz, B., Barber, S., Dzau, J., 2002. Knowledge of stimulus repetition affects the magnitude and spatial distribution of low frequency eventrelated brain potentials. Audiol. Neurootol. 7 (5), 303-314.

Clementz, B., Dzau, J., Blumenfeld, L., Mathews, S., Kissler, J., 2003. Ear of stimulation determines schizophrenia-normal brain activity differences in an auditory paired-stimuli design. Eur. J. Neurosci. 18 (10), 2853-2858.

Clementz, B., Wang, J., Keil, A., 2008. Normal electrocortical facilitation but abnormal target identification during visual sustained attention in schizophrenia. J. of Neurosci. 28 (50), 13,411-13,418.

Cullum, C., Harris, J., Waldo, M., Smernoff, E., Madison, A., Nagamoto, H., et al., 1993. Neurophysiological and neuropsychological evidence for attentional dysfunction in schizophrenia. Schizophr. Res. 10 (2), 131-141.

Deiber, M., Missonnier, P., Bertrand, O., Gold, G., Fazio-Costa, L., Ibanez, V., et al., 2007. Distinction between perceptual and attentional processing in working memory tasks: a study of phase-locked and induced oscillatory brain dynamics. J. Cogn. Neurosci. 19 (1), 158-172.

Erwin, R.J., Turetsky, B.I., Moberg, P., Gur, R.C., Gur, R.E., 1998. P50 abnormalities in schizophrenia: relationship to clinical and neuropsychological indices of attention. Schizophr. Res. 33, 157-167.

Freedman, R., Adler, L.E., Gerhardt, G.A., Waldo, M., Baker, N., Rose, G.M., et al., 1987. Neurobiological studies of sensory gating in schizophrenia. Schizophr. Bull. 13 (4), 669-678.

Gratton, G., Coles, M.G.H., Donchin, E., 1983. A new method for off-line removal of ocular artifact. Electroencephalogr. Clin. Neurophysiol. 55, 468-484.

Guterman, Y., Josiassen, R.C., Bashore, T.R., 1992. Attentional influence on the P50 component of the auditory event-related brain potential. Int. J. Psychophysiol 12,197-209.

Haenschel, C., Baldeweg, T., Croft, R., Whittington, M., Gruzelier, J., 2000. Gamma and beta frequency oscillations in response to novel auditory stimuli: a comparison of human electroencephalogram (EEG) data with in vitro models. Proc. Natl. Acad. Sci. U.S.A. 97 (13), 7645-7650.

Hermann, C.S., Grigutsch, M., \& Busch, N.A., 2005. EEG oscillations and wavelet analysis. In T.C. Handy (Ed.), Event-related potentials: A methods handbook. Cambridge, Massachusetts: The MIT Press.

Hong, L.E., Summerfelt, A., McMahon, R.P., Thaker, G., Buchanan, R., 2004. Gamma/beta oscillation and sensory gating deficit in schizophrenia. NeuroReport 15 (1), 155-159.
Hong, L.E., Buchanan, R.W., Thaker, G.K., Shepard, P.D., Summerfelt, A., 2008. Beta $(16 \mathrm{~Hz})$ frequency neural oscillations mediate auditory sensory gating in humans. Psychophysiology 45, 197-204.

Johannesen, J.K., Kieffaber, P.D., O'Donnell, B.F., Shekhar, A., Evans, J.D., Hetrick, W.P., 2005. Contributions of subtype and spectral frequency analyses to the study of P50 ERP amplitude and suppression in schizophrenia. Schizophr. Res. 78, 269-284.

Johannesen, J., Bodkins, M., O'Donnell, B., Shekhar, A., Hetrick, W., 2008. Perceptual anomalies in schizophrenia co-occur with selective impairments in the gamma frequency component of mid-latency auditory ERPs. J. Abnorm. Psychol. 117 (1), 106-118.

Kisley, M., Cromwell, Z., 2006. Gamma and beta neural activity evoked during a sensory gating paradigm: effects of auditory somatosensory and crossmodal stimulation. Clin. Neurophysiol. 117 (11), 2549-2563.

Klimesch, W., 1999. EEG alpha and theta oscillations reflect cognitive and memory performance: a review and analysis. Brain Res. Rev. 29, 169-195.

Kogoj, A., Pirtosek, Z., Tomori, M., Vodusek, D., 2005. Event-related potentials elicited by distractors in an auditory oddball paradigm in schizophrenia. Psychiatry Res. 137 (1-2), 49-59.

Kopell, N., Ermentrout, G.B., Whittington, M.A., Traub, R.D., 1999. Gamma rhythms and beta rhythms have different synchronization properties. Proc. Natl. Acad. Sci. U.S.A. (4), 1867-1872.

Krishnan, G., Skosnik, P., Vohs, J., Busey, T., O'Donnell, B.F., 2005. Relationship between steady-state and induced gamma activity to motion. NeuroReport 16 (6), 625-630.

Light, G., Geyer, M.A., Clementz, B., Cadenhead, K., Braff, D.L., 2000. Normal P50 suppression in schizophrenia patients treated with atypical antipsychotic medications. Am. J. Psychiatry 157 (5), 767-771.

Palva, S., Palva, J.M., 2007. New vistas for a-frequency band oscillations. Trends Neurosci. 30 (4), 150-158.

Pantev, C., Makeig, S., Hoke, M., Galambos, R., Hampson, S., Gallen, C., 1991. Human auditory evoked gamma-band magnetic fields. Proc. Natl. Acad. Sci. U.S.A. 88, 8996-9000.

Patterson, J.V., Hetrick, W.P., Boutros, N.N., Jin, Y., Sandman, C., Stern, H., et al., 2008. P50 sensory gating ratios in schizophrenics and controls: a review and data analysis. Psychiatry Res. 158 (2), 226-247.

Pesonen, M., Hamalainen, H., Krause, C., 2007. Brain oscillatory 4-30 Hz responses during a visual $\mathrm{N}$-back memory task with varying memory load. Brain Res. 1138 (17), 1-7.

Schack, B., Klimesch, W., Sauseng, P., 2005. Phase synchronization between theta and upper alpha oscillations in a working memory task. Int. J. Psychophysiol. 57, 105-114.

Smith, D.A., Boutros, N.N., Schwarzkopf, S.B., 1994. Reliability of P50 auditory event-related potential indices of sensory gating. Psychophysiology 31 , 495-502.

Tallon-Baudry, C., Bertrand, O., 1999. Oscillatory gamma activity in humans and its role in object representation. Trends Cogn. Sci. 3 (4), 151-162.

Thoma, R., Hanlon, F., Moses, S., Edgar, J., Huang, M., Weisend, M., et al., 2003. Lateralization of auditory sensory gating and neuropsychological dysfunction in schizophrenia. Am. J. Psychiatry 160 (9), 1595-1605.

Traub, R.D., Whittington, M., Buhl, E., Jefferys, J., Faulkner, H., 1999. On the mechanism of the gamma $\rightarrow$ beta frequency shift in neuronal oscillations induced in the rat hippocampal slices by tetanic stimulation. J. Neurosci. 19 (3), 1088-1105.

Trautner, P., Rosburg, T., Dietl, T., Fell, J., Korzyukov, O.A., Kurthen, M., et al., 2006. Sensory gating of auditory evoked and induced gamma band activity in intracranial recordings. NeuroImage 32, 790-798.

Whittington, M.A., Traub, R., Faulkner, H., Stanford, I., Jefferys, J., 1997. Recurrent excitatory postsynaptic potentials induced by synchronized fast cortical oscillations. Proc. Natl. Acad. Sci. U.S.A. 94 (22), $12,198-12,203$.

Woldorff, M.G., Gallen, C.C., Hampson, S.A., Hillyard, S.A., Pantev, C., Sobel, D., et al., 1993. Modulation of early sensory processing in human auditory cortex during auditory selective attention. Proc. Natl. Acad. Sci. U.S.A. 90 (18), 8722-8726. 